\title{
Novel Eye Plaque Designs for Brachytherapy of Iris and Ciliary Body Melanoma and the First Clinical Application
}

\author{
Wu Liu ${ }^{a, d}$ Jenna May Kim ${ }^{b}$ Benjamin K. Young ${ }^{b}$ Ravinder Nath ${ }^{a} d$ \\ Zhe Chen $^{a, d}$ Roy H. Decker ${ }^{a} d$ Melvin A. Astrahan ${ }^{c}$ \\ Renelle Pointdujour-Lim ${ }^{b}$ d \\ a Department of Therapeutic Radiology, Yale University School of Medicine, New Haven, CT, USA; bepartment of

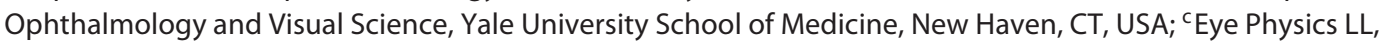 \\ Los Alamitos, CA, USA; ${ }^{d}$ Yale Smilow Cancer Center, Yale University School of Medicine, New Haven, CT, USA
}

\section{Keywords}

Melanoma $\cdot$ Brachytherapy $\cdot$ Eye plaque $\cdot$ Iris-ciliary body

\begin{abstract}
Background: While traditional eye plaque brachytherapy can be used for the treatment of iris melanoma, it faces challenges of poor patient tolerability due to cornea-plaque touch caused by radius of curvature mismatch and potential dosimetric inaccuracy from incomplete coverage. We present novel plaque designs and the first clinical application of the plaques for iris melanoma. Methods: Two dome-shaped plaques (EP2132 and EP1930) were designed to vault above the cornea to treat tumors of the iris and ciliary body. Imagebased treatment planning of the first 2 clinical cases using the EP2132 plaque covered the tumor base plus a $2 \mathrm{~mm}$ margin and the involved ciliary body with at least $75 \mathrm{~Gy}$ to the tumor apex. Results: The tumors decreased in size following treatment. The patients tolerated the treatment well. There was no adverse event associated with the traditional iris plaques, such as decreased vision, pain, corneal edema, glaucoma, or cataract. Conclusion: The novel dome-shaped
\end{abstract}

(c) 2018 S. Karger AG, Basel

E-Mail karger@karger.com

www.karger.com/oop plaques for the treatment of iris melanoma provide effective dose distribution, improved surgical maneuverability, and increased tolerability for the patient. This plaque model can be used to treat iris melanoma of various sizes, configurations, and locations, including the ciliary body. The need for a customized plaque platform for each patient is minimized.

(c) 2018 S. Karger AG, Basel

\section{Introduction}

Iris melanoma is a subtype of uveal melanoma historically thought to have a more indolent natural course compared to melanoma of the ciliary body or choroid [1]. Before 2001, the metastatic rate of iris melanoma was reported to range from $3-5 \%$ over 10 years $[1,2]$. More recent studies suggest a higher rate of metastasis. A multicenter study from 2012, for example, reported a rate of $10.7 \%$ at 5 years [3], and a single-center study of the same year revealed rates of 9 and $11 \%$ at 10 and 20 years, respectively [4]. The management of iris melanoma has accordingly shifted towards intervention rather than obser- 
Fig. 1. Two novel plaques designed for the treatment of iris melanoma. a The EP2132 plaque is circular with a diameter of $21 \mathrm{~mm}$ and an opening of $5 \mathrm{~mm}$ at the center. It has 32 seed slots in a radial pattern centrally and concentric peripherally. b The EP1930 plaque is slightly smaller than EP2132 with $19 \mathrm{~mm}$ in diameter and a $2-\mathrm{mm}$ opening at the center. There are 30 concentric seed slots in EP1930. The brown lines indicate seed slots. Note the placement of the eyelets, 4 clock hours apart, for both plaques. The color refers to the online version only.
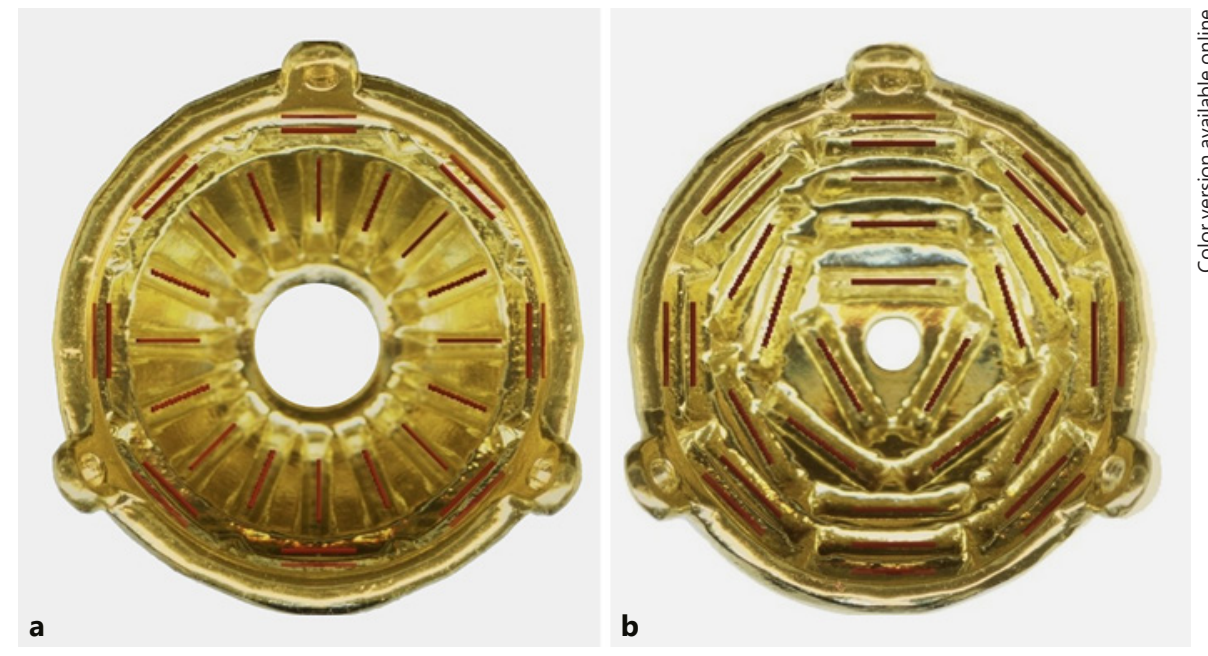

vation. Treatment options have traditionally included local resection, plaque brachytherapy $(\mathrm{PB})$, proton beam radiation therapy, and enucleation. PB is now widely used [5-9] due to its minimally invasive nature compared to resection or enucleation $[7,10]$. Compared to proton therapy, $\mathrm{PB}$ delivers less radiation to the orbit and remainder of the globe [11] and leads to fewer post-treatment glaucoma [12-14]. Therefore, except for very large tumors, $\mathrm{PB}$ has been the treatment of choice over proton beam therapy for many ocular oncologists [15].

Nonetheless, there are challenges related to treating iris melanoma with $\mathrm{PB}$, including plaque-corneal contact and resultant patient discomfort during treatment, uncertain plaque placement and possibly incomplete dosimetric coverage, and the potential need for plaque customization for each patient. Because the curvature of a standard plaque for choroidal melanoma is usually designed to conform to the sclera, the use of such standard plaques causes cornea-plaque touch and excessive pressure on the cornea $[7,16]$. Finger [17] introduced the use of a $0.1-\mathrm{mm}$-thick amniotic membrane that is placed between the plaque and the cornea to reduce patient discomfort. Custom plaques [11] and modified Collaborative Ocular Melanoma Study (COMS) plaques [18] with boomerang, partial-ring, or fully-circular shapes for individual patients have been used to improve the treatment of iris melanoma. However, they may still maintain the curvature mismatch, leading to patient discomfort, and they may not be ideal when treating tumors extending to the visual axis or when full coverage of the entire region under the entire cornea is indicated. A modified COMS plaque without the central hole that provides full cover- age has been reported [19] by machining the original COMS silastic insert to fit the cornea curvature. It requires patient-specific custom machinery, which may not be practical at all institutions.

In this study, we report 2 novel designs of domeshaped plaques (Eye Physics model EP2132 and EP1930) aimed at solving the problem of unpredictable comfort, coverage, and cost. These new plaques vault over the cornea to treat tumors of the iris and ciliary body with up to $360^{\circ}$ uniform dosimetric coverage. They provide more seed slots than COMS plaques. The potential seed arrangement permutation offers flexible tumor coverage and eliminates the need for custom hardware for each patient. The first clinical cases, surgical method, and outcome using the EP2132 plaque with image-based treatment planning are presented.

\section{Materials and Methods}

Two dome-shaped plaques, EP2132 (Fig. 1a) and EP1930 (Fig. 1b), were designed using Plaque Simulator Version 6 (Eye Physics LLC, Los Alamitos, CA, USA), exported to a stereolithograpy (.stl) file and prototyped using a Formlabs 3D printer (Somerville, MA, USA) with a 0.001 -inch resolution and direct castable blue resin. The castable resin prototype was then used to produce the plaque in 18-karat gold with better than $0.2-\mathrm{mm}$ accuracy. The core design for the seed slots is the same as for other Eye Physics plaques. EP2132 and EP1930 each features 3 suture eyelets $120^{\circ}$ apart for improved stability and accuracy when suturing to the sclera.

The EP2132 plaque is circular with a diameter of $21 \mathrm{~mm}$ with a 5 -mm opening at the center for cornea oxygenation. The thickness of the plaque is $2 \mathrm{~mm}$. The central hole lies in axial alignment with the pupil. The plaque has 32 seed slots in a radial pattern cen- 
trally and a circular pattern in the periphery for flexible dose coverage of the iris and ciliary body. The EP1930 plaque is circular with a diameter of $19 \mathrm{~mm}$ and an opening of $2 \mathrm{~mm}$ at the center. Thirty seed slots lie in a concentric circular pattern. The seed patterns of the plaques are intended for different size eyes and dose coverage anterior and posterior to the limbus. EP1930 provides more seed slots for anterior coverage. The central opening size is as large as practical for each seed pattern.
The radius of curvature of the novel plaques is $9 \mathrm{~mm}$, which contrasts with that of COMS plaques that is $12.3 \mathrm{~mm}$. It is the reduced radius of curvature of the new plaques combined with the large diameter of $19 \mathrm{~mm}$ (EP1930) or $21 \mathrm{~mm}$ (EP2132) that creates the vault between the plaque and the ocular surface. For comparison, the radius of curvature of the cornea is $7.5 \mathrm{~mm}$, and the corneal diameter is $12 \mathrm{~mm}$.
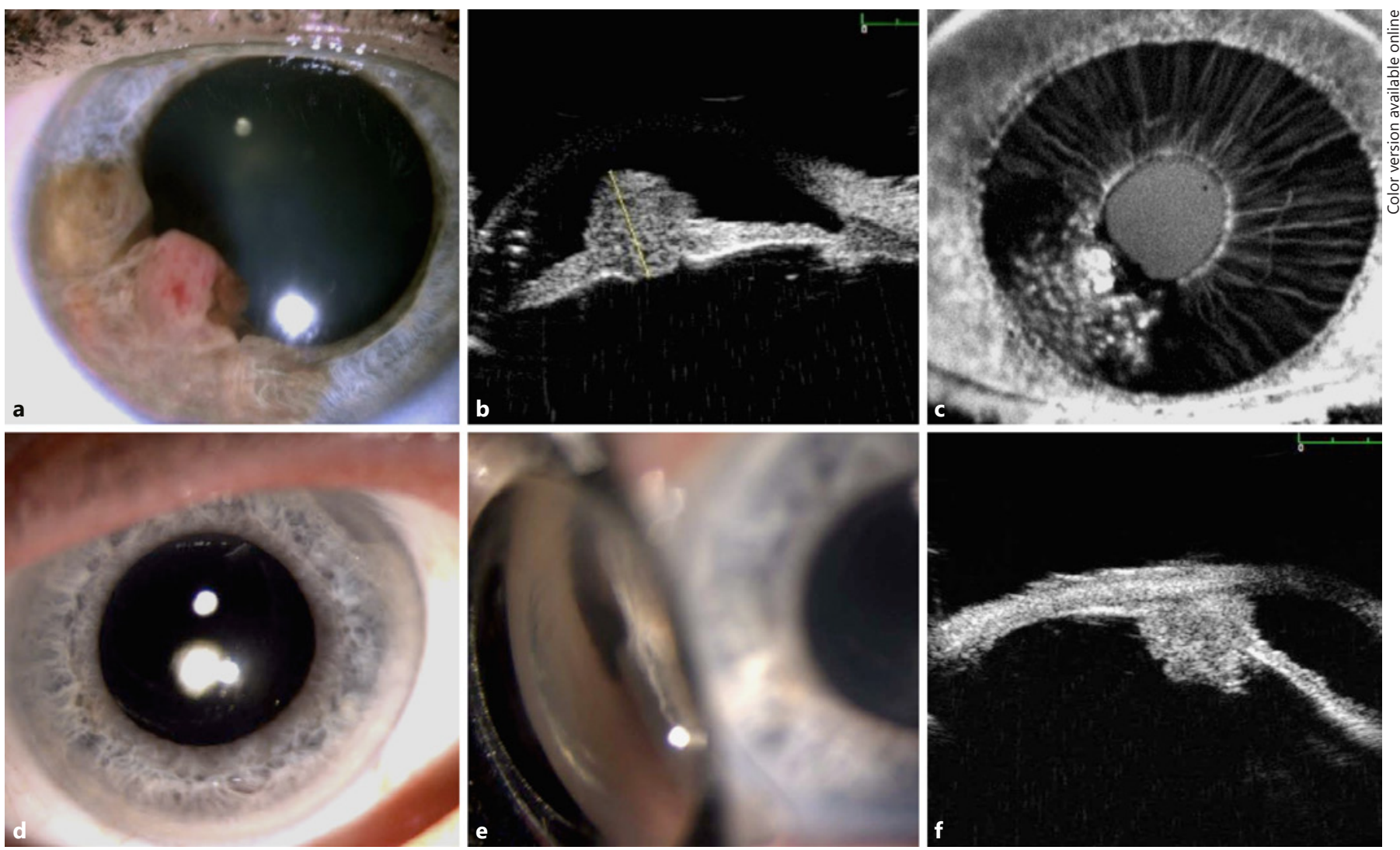

Fig. 2. Multimodal imaging of the iris melanoma in Case $1(\mathbf{a}-\mathbf{c})$ and Case $2(\mathbf{d}-\mathbf{f})$. a Slit-lamp photograph shows the inferotemporal iris melanoma with a central nodule that extends into the pupillary axis. b High-frequency ultrasound biomicroscopy demonstrates the axial height of the tumor. c Anterior segment fluores- cein angiography shows leakage of fluorescein in the vessels associated with the iris melanoma. d Slit-lamp photograph of Case 2 showing iridociliary melanoma in the superotemporal quadrant. e Gonioscopy shows the location of the tumor deep in the angle. f B-mode ultrasonography shows the ciliary body involvement.
Fig. 3. Pretreatment planning for Case $1(\mathbf{a}-\mathbf{c})$ and Case $2(\mathbf{d}-\mathbf{f})$. a The retinal diagram demonstrates an overlaid slit-lamp photograph, the plaque, seed placement pattern, and isodose lines. The tumor outline is delineated by the brown contour. The seeds are shown as light blue bars. The thick cyan isodose line indicates the coverage by the prescribed 75 Gy. b A dose-area histogram on the retinal spherical surface demonstrates the coverage of the tumor base plus margin and the doses to the critical retinal struc- tures. c A dose-volume histogram shows 3D dosimetry to the tumor, lens, and the entire eye. d Isodose map for Case 2 with an overlaid slit-lamp photograph. The majority of the iridociliary melanoma is posterior to the limbus. e Tumor-meridian view of the eye with the EP2132 plaque. f Tumor-meridian view of the eye with the COMS22 plaque showing substantial overlap of the plaque's seed carrier with the cornea. Colors refer to the online version only.

(For figure see next page.)
Liu/Kim/Young/Nath/Chen/Decker/ Astrahan/Pointdujour-Lim 


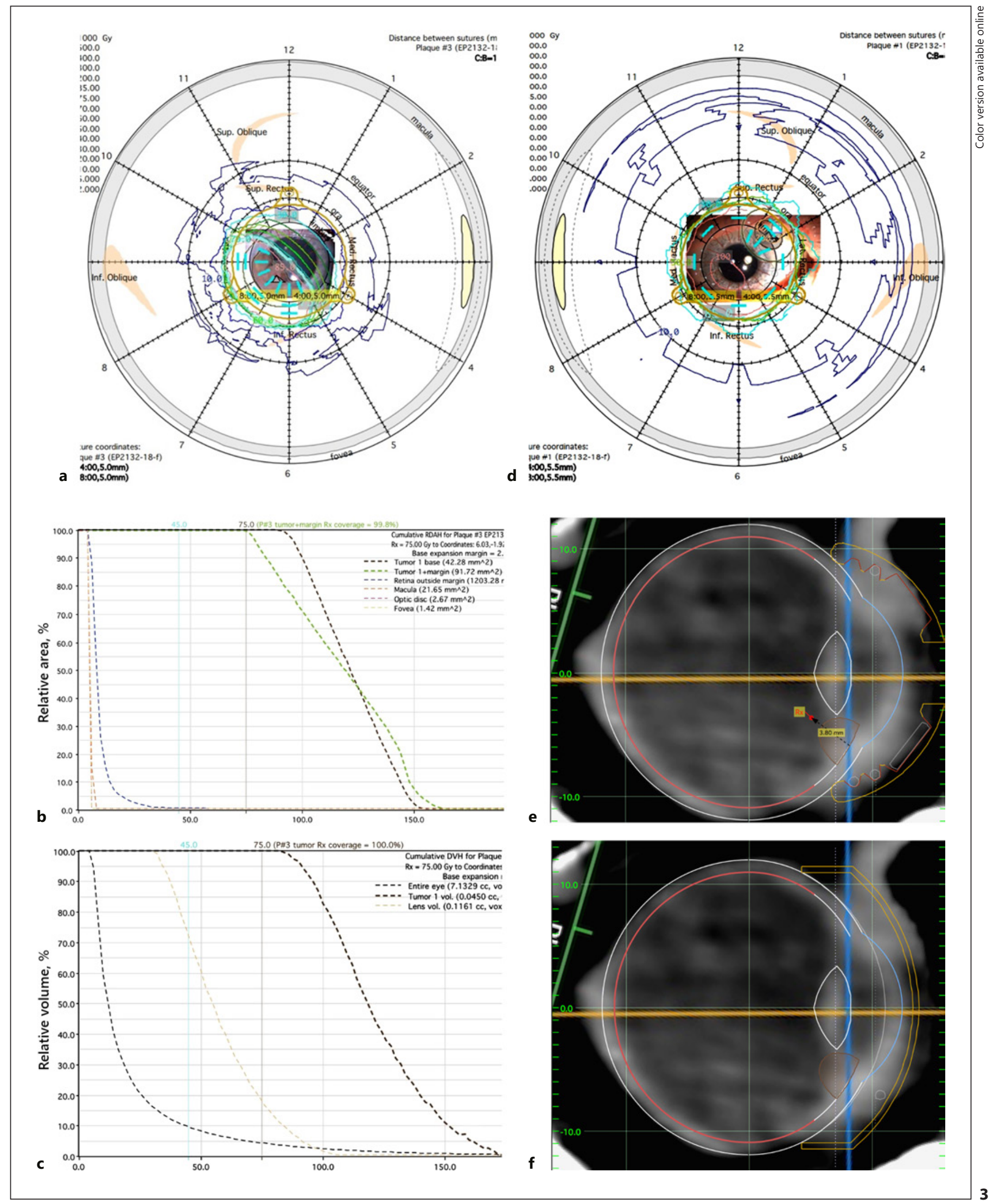

Novel Plaque for Iris Melanoma

Ocul Oncol Pathol 2019;5:220-227

DOI: $10.1159 / 000493269$ 

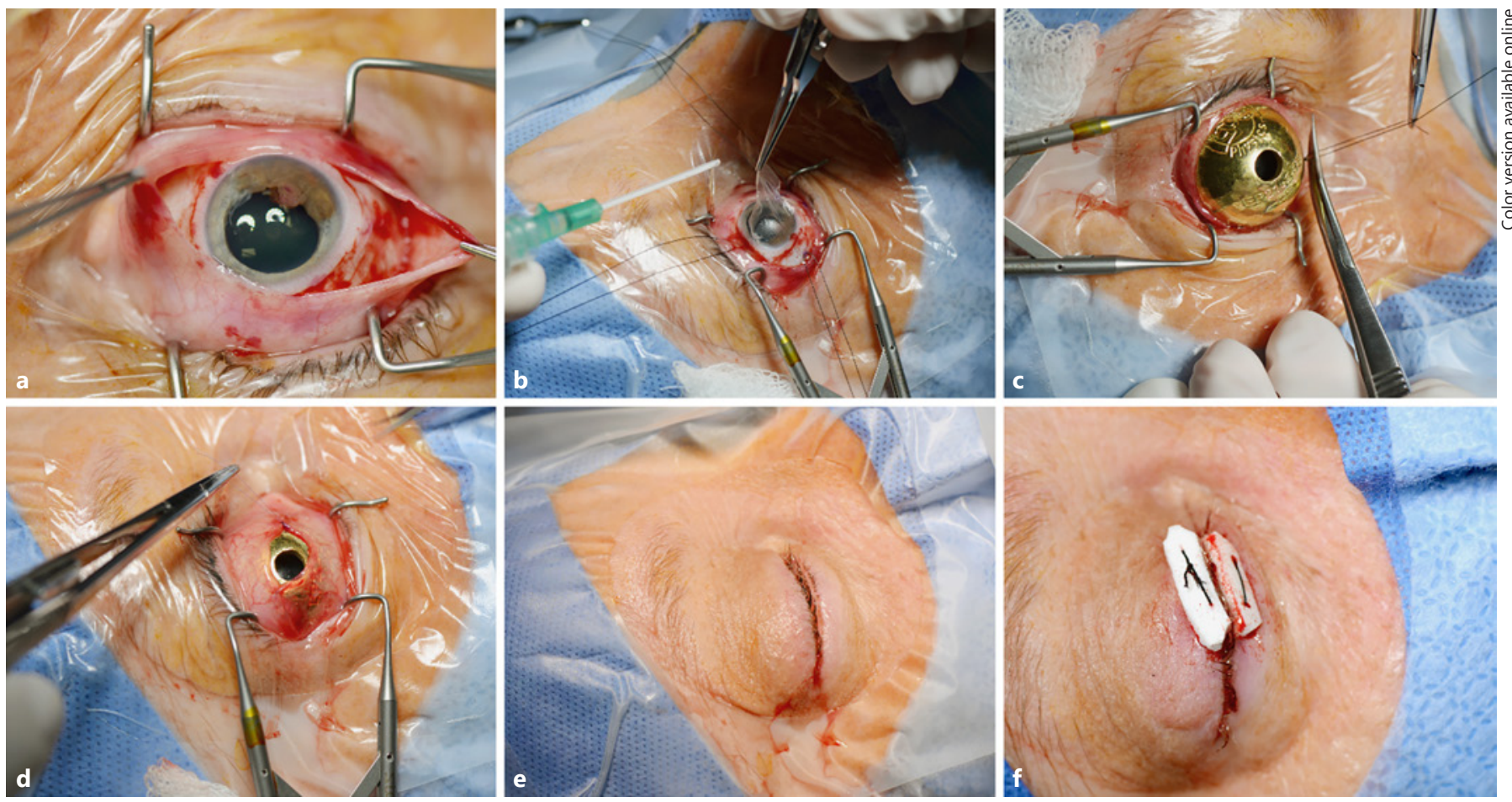

Fig. 4. Intraoperative photographs illustrating the procedure of implanting the plaque covering the cornea. After $360^{\circ}$ of perilimbal peritomy (a), amniotic membrane was placed over the cornea (b). The plaque was placed over the cornea and then sutured into place

using the 3 eyelets (c). The conjunctiva was draped over the plaque and then suture closed (d). There was no tension on the eyelids upon closure (e). Temporary tarsorrhaphy of the eyelids was performed (f).

\section{Case Presentations}

Case 1

A 49-year-old Caucasian female with an iris nevus of the right eye was observed since 2012. The initial measurements were $8 \times$ $3.8 \times 1.6 \mathrm{~mm}$. She developed recurrent episodes of hyphema associated with decreased vision 4 years later. Upon initial evaluation by an ocular oncologist, the mass was noted to have grown, measuring $8 \times 4 \times 3 \mathrm{~mm}$ (Fig. $2 \mathrm{a}$ ). The central nodule of the melanoma had doubled in size in the 4-year time frame and extended medially into the pupillary axis (Fig. 2b). Iris fluorescein angiography showed diffuse, multifocal hyperfluorescence and late leakage (Fig. 2c).

Plaque planning involved the use of computed tomography imaging to construct a patient-specific eye model [20]. The tumor was contoured using slit-lamp photography (Fig. 3a). Three-dimensional treatment planning was performed using Plaque Simulator version 6.5 for EP2132. The brown contour delineated the tumor. Thirteen Iodine-125 seeds were used to deliver therapeutic radiation to the tumor. With the suture eyelets of the plaque placed at 4,8 , and 12 o'clock in the perilimbal sclera $5.0 \mathrm{~mm}$ from the limbus, 7 seeds in the center of the plaque covered the tumor, and 6 seeds in the plaque periphery covered the ciliary body. The seeds are demonstrated as light blue bars in Figure $3 \mathrm{a}$ (the color refers to the online version only).

The dose calculations were performed using a linear source model with anisotropy correction according to AAPM TG43 [21]. Slot collimation was enabled in the Plaque Simulator software [22], which activated ray tracing of the collimation of primary radiation by the rectangular slot surrounding each seed. Partial exposure was enabled, which calculated the fraction of primary radiation from a linear source that does not pass through the shell. The doses were calculated under the infinite homogeneous water assumption according to AAPM TG129 [23]. Figures 3b and c show the dose area histogram on the retinal spherical surface and the dose volume histogram, respectively. The planned dose to the tumor apex was 85 Gy. At least 75 Gy covered the tumor base with an additional margin of $2 \mathrm{~mm}$. The doses to the cornea apex, center of the crystalline lens, center of the optic disc, and fovea were 94, 57, 6, and $6 \mathrm{~Gy}$, respectively. About half of the cornea received over $75 \mathrm{~Gy}$, but the dose to the superonasal sector of the cornea - the quadrant farthest from the tumor - was gradually reduced to $30 \mathrm{~Gy}$.

Figure 4 details the surgical procedure of plaque application. A 360-degree limbal peritomy was made, and Tenon's capsule was carefully dissected in all quadrants to reveal the bare sclera around the corneal limbus (Fig. 4a). Amniotic membrane was applied to the cornea with tissue glue for corneal protection (Fig. 4b). The plaque was placed over the eye with the base of the plaque in contact with the bare sclera around the cornea (Fig. 4c). Using a 5-0 nylon suture, the eyelets were sutured to the sclera in the preplanned orientation. The plaque did not have contact with the cor- 

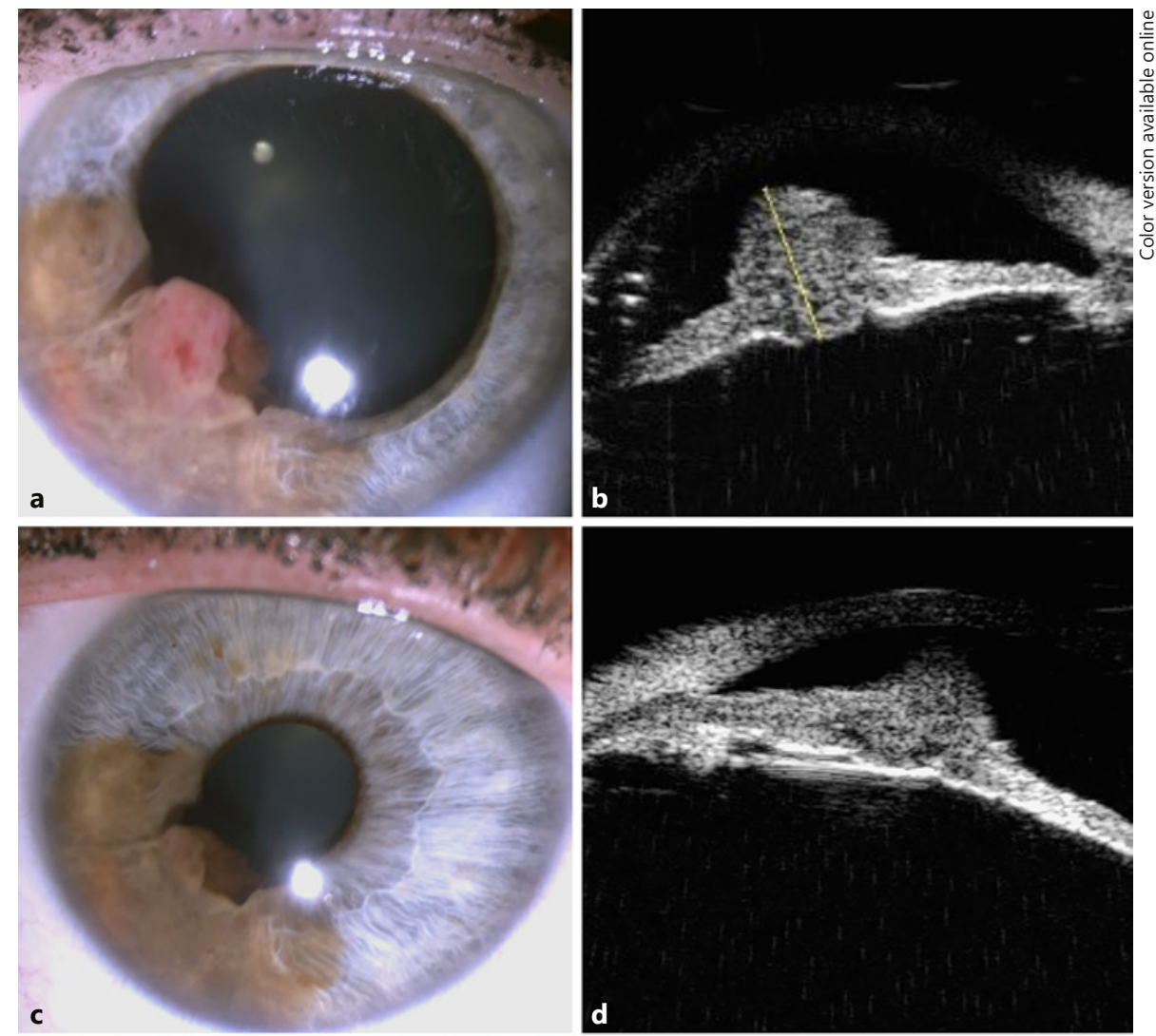

Fig. 5. Side-by-side view of the iris melanoma cases before and after PB in Case 1 (a-d) and Case 2 (e, f). a The inferotemporal iris melanoma had a highly vascular core that dramatically reduced in size after PB (b). High-frequency B-mode ultrasonography prior to $\mathrm{PB}$ demonstrates a reduction in the axial height of the tumor's central core from $3 \mathrm{~mm}$ (c) to $1.8 \mathrm{~mm}$ at 4 months from brachytherapy (d). e The prePB slit-lamp photograph of the iridociliary melanoma. f One month after PB, the tumor appears smaller and less pigmented.
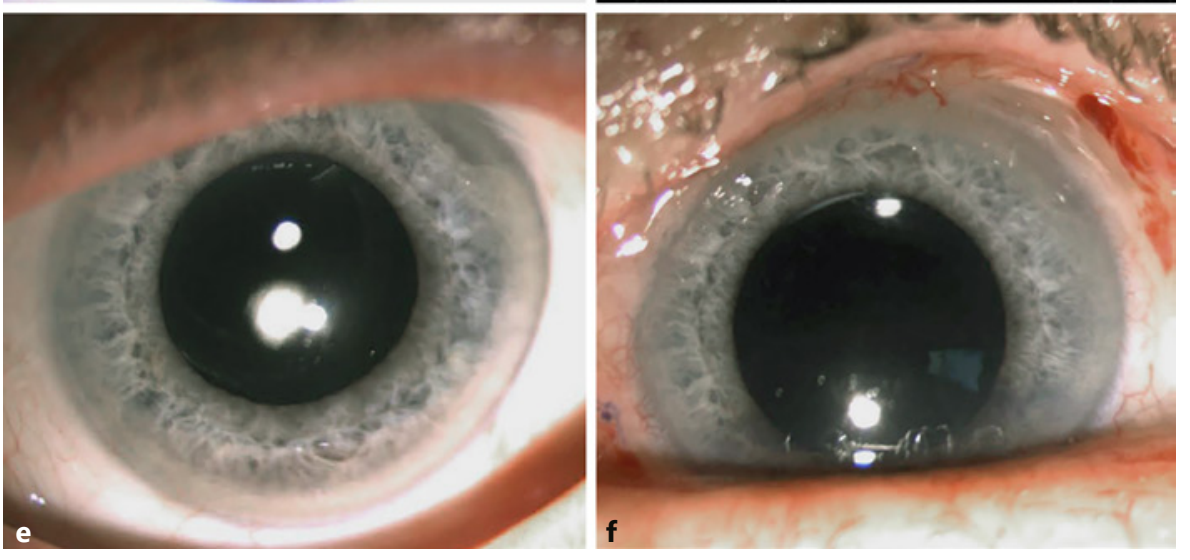

nea. A Gunderson flap was fashioned by completely draping the conjunctiva over the plaque (Fig. $4 \mathrm{~d}$ ). At the end of the procedure, the eyelids easily came together without tension (Fig. 4e). The eyelids were surgically closed by placing a temporary tarsorrhaphy with bolsters (Fig. 4f). Four days later, the tarsorrhaphy was taken down and the plaque was surgically removed.

\section{Case 2}

A 72-year-old male with superotemporal iridociliary melanoma measuring $3.5 \times 3 \times 3 \mathrm{~mm}$ (Fig. $2 \mathrm{~d}-\mathrm{f}$ ) underwent PB using EP2132. The dose calculation and plaque planning were performed using identical methods as in Case 1. Figure $3 \mathrm{~d}$ shows the isodose map overlaid with the slip-lamp photograph. Most of the tumor was posterior to the limbus, and the entire limbus was included in the treatment due to angle seeding of the tumor. Eight seeds were placed on the inner circle of the peripheral seed slots of the plaque to cover the entire limbus, and 4 additional seeds ( 3 anterior and 1 posterior) were placed at 12 to 3 clock hours to ensure tumor coverage with $85 \mathrm{~Gy}$ reaching $>4 \mathrm{~mm}$ posterior to the limbus. Figures $3 \mathrm{e}$ and $\mathrm{f}$ show the tumormeridian view of the eye with the EP2132 and COMS22 plaques, respectively. EP2132 ensures full-dose coverage without plaque-cornea contact. It is, however, impractical to plan accurately with COMS22 due to the significant overlap of the plaque's seed carrier with the cornea. The surgical method of plaque implantation was identical to that of Case 1, although amniotic membrane was not applied. 


\section{Results}

\section{Case 1}

The patient reported minimal discomfort during the implantation period of 4 days. Ophthalmic examinations performed at 14 days and 4 months after plaque implantation revealed absence of secondary neovascularization of the iris or angle, corneal edema, keratopathy, posterior synechiae, or cataract. There was decreased vascularity of the tumor surface and no recurrence of hyphema. The tumor nodule had decreased from the pre-PB (Fig. 5a, b) height of 3.0-1.8 mm after treatment (Fig. 5c, d). The patient's preoperative distance visual acuity was 20/20 and $20 / 25$ at 4 months following PB. She continues to be monitored for potential radiation- and tumor-associated adverse effects and undergoes routine systemic metastasis surveillance.

\section{Case 2}

The patient reported minimal discomfort during the treatment period of 4 days. At the time of plaque removal, the patient was noted to have corneal epithelial defect. He was prescribed erythromycin. At the 1-month follow up, his best-corrected vision was 20/40 - compared to his preoperative vision of 20/30, and the corneal epithelial defect had resolved. The tumor decreased from pre-PB dimensions of $3.5 \times 3$ to $3 \times 1 \mathrm{~mm}$ (Fig. $5 \mathrm{e}, \mathrm{f}$ ). There were no radiation-associated adverse effects.

\section{Discussion}

For the treatment of iris or iridociliary melanoma, the novel plaques were specifically designed with a smaller radius of curvature than that of the conventional COMS and EP plaques, while maintaining a large diameter. Such dimensions preclude contact between the plaque and cornea, improving patient comfort, plaque positioning accuracy, and plaque stability during the treatment period. This design allows for a uniform surgical approach and decreases the demand for resources required to produce customized plaque for each patient, hence minimizing cost. The new designs also provide more seed placement options due to their hemispheric configuration rather than a simple two-dimensional ring around the limbus as seen in modified COMS plaques. They therefore offer up to $360^{\circ}$ coverage of the anterior segment between the cornea and lens and near the corneal limbus, broadening their application for nearly any size and anatomic location of iris or iridociliary melanoma.
Additionally, in contrast to the COMS plaques, the EP plaques do not use a separate seed carrier and thus eliminate seed placement uncertainty that comes with mobile and flexible seed carriers. The seed slots built into the EP plaques are designed to collimate each radiation source, effectively shielding laterally directed primary radiation that does not contribute to tumor treatment. Therefore, the plaques are overall thinner than COMS plaques and yield a more homogenous dose distribution between the plaque and tumor apex in addition to the reduction in the radiation dose beyond the target.

The central opening of each plaque improves oxygenation of the cornea and thus preserves the integrity of the cornea throughout treatment. Notable differences between the two plaques include the size of the central opening and the overall diameter. EP1930 with the smaller opening allows for more coverage of the anterior and central tumor, but its smaller overall size may be more desirable in an eye with limited conjunctival laxity for the construction of Gunderson flap during plaque application. In contrast, the larger EP2132 is more appropriate for tumors involving the ciliary body. The combination of the dosimetric need for the target tumor and the conjunctival laxity of the recipient eye may ultimately dictate the choice of one plaque over another. Despite the slight differences, the surgical technique for the application of the two plaques is identical. The use of amniotic membrane on the cornea is optional, as demonstrated by Cases 1 and 2 that had the same postoperative vision and corneal surface outcomes.

One potential for dosimetric error of the new plaque designs comes from air gaps that can form between the plaque and cornea. Because the inverse-square law rather than attenuation is dosimetrically dominant at short distances from the seeds, however, accounting for the air gap is clinically insignificant in dosimetry compared to assuming overall homogeneous water. Our calculations show that the dose difference is within $1.5 \%$ when accounting for the air gap versus assuming the air gap as water.

In summary, the novel plaque designs described herein offer several advantages over COMS or modified COMS plaques - comfort for the patient, coverage of the tumor, cost minimization, broad applicability, and uniformity of the surgical method. More outcome studies are needed to elucidate the efficacy and tumor control with the new plaque design in the treatment of iris melanoma. 


\section{Statement of Ethics}

The photographs of the patient were taken after obtaining written informed consent from the patient. The provisions of the Declaration of Helsinki for research involving human subjects were followed. No animal research was conducted as part of this work.

\section{Disclosure Statement}

This investigation was partly supported by NIH Research Grant CA 16359 from the National Cancer Institute. The senior authors are members of the Yale Cancer Center. Melvin Astrahan, $\mathrm{PhD}$, has the sole proprietorship of Eye Physics LLC and the Plaque Simulator software.

\section{References}

1 Shields CL, Shields JA, Materin M, Gershenbaum E, Singh AD, Smith A. Iris melanoma: risk factors for metastasis in 169 consecutive patients [published online ahead of print 2001/01/11]. Ophthalmology. 2001 Jan; 108(1):172-8

2 Geisse LJ, Robertson DM. Iris melanomas [published online ahead of print 1985/06/15]. Am J Ophthalmol. 1985 Jun;99(6):638-48.

3 Khan S, Finger PT, Yu GP, Razzaq L, Jager MJ, de Keizer RJ, et al. Clinical and pathologic characteristics of biopsy-proven iris melanoma: a multicenter international study [published online ahead of print 2011/09/14]. Arch Ophthalmol. 2012 Jan;130(1):57-64.

4 Shields CL, Kaliki S, Shah SU, Luo W, Furuta $\mathrm{M}$, Shields JA. Iris melanoma: features and prognosis in 317 children and adults [published online ahead of print 2012/03/01]. J AAPOS. 2012 Feb;16(1):10-6.

5 Chaugule SS, Finger PT. Regression Patterns of Iris Melanoma after Palladium-103 (103Pd) Plaque Brachytherapy [published online ahead of print 2017/04/06]. Ophthalmology. 2017 Jul;124(7):1023-30.

6 Shields CL, Shah SU, Bianciotto CG, Emrich J, Komarnicky L, Shields JA. Iris melanoma management with iodine-125 plaque radiotherapy in 144 patients: impact of melanomarelated glaucoma on outcomes [published online ahead of print 2012/09/18]. Ophthalmology. 2013 Jan;120(1):55-61.

7 Finger PT. Plaque radiation therapy for malignant melanoma of the iris and ciliary body [published online ahead of print 2001/09/01]. Am J Ophthalmol. 2001 Sep;132(3):328-35.

8 Shields CL, Naseripour M, Shields JA, Freire J, Cater J. Custom-designed plaque radiotherapy for nonresectable iris melanoma in $38 \mathrm{pa}-$ tients: tumor control and ocular complications [published online ahead of print 2003/04/30]. Am J Ophthalmol. 2003 May; 135(5):648-56.
9 Yousef YA, Finger PT. Lack of radiation maculopathy after palladium-103 plaque radiotherapy for iris melanoma [published online ahead of print 2011/12/17]. Int J Radiat Oncol Biol Phys. 2012 Jul;83(4):1107-12.

10 Marigo FA, Finger PT. Anterior segment tumors: current concepts and innovations [published online ahead of print 2003/11/12]. Surv Ophthalmol. 2003 Nov-Dec;48(6):56993.

11 Shields CL, Shields JA, De Potter P, Singh AD, Hernandez C, Brady LW. Treatment of nonresectable malignant iris tumours with custom designed plaque radiotherapy [published online ahead of print 1995/04/01]. Br J Ophthalmol. 1995 Apr;79(4):306-12.

12 Willerding GD, Cordini D, Hackl C, Karle B, Lakotka N, Foerster MH, et al. Proton beam radiotherapy of diffuse iris melanoma in 54 patients [published online ahead of print 2014/12/17]. Br J Ophthalmol. 2015 Jun; 99(6):812-6.

13 Konstantinidis L, Roberts D, Errington RD, Kacperek A, Damato B. Whole anterior segment proton beam radiotherapy for diffuse iris melanoma [published online ahead of print 2013/01/10]. Br J Ophthalmol. 2013 Apr;97(4):471-4.

14 Popovic M, Ahmed II, DiGiovanni J, Shields CL. Radiotherapeutic and surgical management of iris melanoma: A review [published online ahead of print 2017/01/08]. Surv Ophthalmol. 2017 May - Jun;62(3):302-11.

15 Collaborative Ocular Melanoma Study Group. The COMS randomized trial of iodine 125 brachytherapy for choroidal melanoma: V. Twelve-year mortality rates and prognostic factors: COMS report No. 28. Arch Ophthalmol. 2006 Dec;124(12):1684-93.

16 Petousis V, Finger PT, Milman T. Multifocal iris melanoma treated with total anterior segment palladium-103 plaque radiation therapy. Graefes Arch Clin Exp Ophthalmol. 2011 Jun;249(6):937-40.
17 Finger PT. Finger's amniotic membrane buffer technique: protecting the cornea during radiation plaque therapy [published online ahead of print 2008/04/17]. Arch Ophthalmol. 2008 Apr;126(4):531-4.

18 Thomson RM, Furutani KM, Pulido JS, Stafford SL, Rogers DW. Modified COMS plaques for $125 \mathrm{I}$ and 103Pd iris melanoma brachytherapy [published online ahead of print 2010/05/18]. Int J Radiat Oncol Biol Phys. 2010 Nov;78(4):1261-9.

19 Scanderbeg DJ, Vasudev D, Rice RK, Goldbaum M, Mundt AJ. A modified COMS plaque for iris melanoma [published online ahead of print 2011/09/01]. J Contemp Brachytherapy. 2011 Sep;3(3):131-3.

20 Tien CJ, Astrahan MA, Kim JM, Materin M, Chen Z, Nath R, et al. Incorporating patientspecific CT-based ophthalmic anatomy in modeling iodine- 125 eye plaque brachytherapy dose distributions [published online ahead of print 2017/08/06]. Brachytherapy. 2017 Sep - Oct;16(5):1057-64.

21 Nath R, Anderson LL, Luxton G, Weaver KA, Williamson JF, Meigooni AS; American Association of Physicists in Medicine. Dosimetry of interstitial brachytherapy sources: recommendations of the AAPM Radiation Therapy Committee Task Group No. 43. Med Phys. 1995 Feb;22(2):209-34.

22 Astrahan MA. Improved treatment planning for COMS eye plaques [published online ahead of print 2005/03/09]. Int J Radiat Oncol Biol Phys. 2005 Mar;61(4):1227-42.

23 Chiu-Tsao ST, Astrahan MA, Finger PT, Followill DS, Meigooni AS, Melhus CS, et al. Dosimetry of (125)I and (103)Pd COMS eye plaques for intraocular tumors: report of Task Group 129 by the AAPM and ABS [published online ahead of print 2012/10/09]. Med Phys. 2012 Oct;39(10):6161-84. 